\title{
Enhanced Color Flow Imaging of Breast Cancer Vasculature: Continuous Wave Doppler and Three-Dimensional Display
}

\author{
P.L. Carson, PhD, D.D. Adler, MD, J.B. Fowlkes, PhD, K. Harnist, MD, J. Rubin, MD, PhD
}

Two methods of potentially improving the detection and assessment of breast cancer vasculature by color flow Doppler ultrasonography were studied. Use of continuous wave $(\mathrm{CW})$ Doppler imaging was one method evaluated by a comparison of system sensitivity to small vessel flow by continuous wave and pulsed Doppler methods. The second technique demonstrated color flow image acquisition and three-dimensional (3D) display. Six breast cancer patients were examined with both a color flow pulsed system and a CW Doppler system employing a hand-held transmitter-receiver pair with crossed-beam patterns. The $\mathrm{CW}$ unit consistently revealed more regions of tumor flow and multidirectional flow. Good 3D displays were achieved on larger pulsatile vessels, from images obtained during systole and selected for minimal noise. KEY WORDS: Ultrasound, Doppler studies; Breast, ultrasound studies; Breast neoplasms.
A n evaluation of tumor blood flow characteristics is usually an important, although not definitive, component of cancer diagnosis. This is true in computed tomography (CT), during which large amounts of simple iodinated contrast agents are injected for most examinations related to soft tissue neoplasia. Information is obtained about the vascularity, necrosis, and, possibly, leaky vessels, and this usually gives an adequate basis for a decision on whether to perform a biopsy. It is clear that vascularity related properties are diagnostic indicators, but questions remain about their value when weighed against the costs and risks of measurement techniques. This is particularly true in the breast, where minimal tissue penetration requirements and the high tumor contrast usually provided by calcifications and fatty tissues make mammography relatively effective among

Received from the Department of Radiology, University of Michigan Medical Center, Ann Arbor, MI 48109-0553.

Address correspondence and reprint requests to Paul L. Carson, PhD, Department of Radiology, University of Michigan Medical Center, Kresge III, Room 3324, Ann Arbor ${ }_{f}$ MI 48109-0553.

The $C W$-pulsed wave comparison was supported in part by an American Cancer Society Clinical Oncology Career Development Award. noncontrast radiography procedures. Previous work on angiography of carcinoma of the breast ${ }^{1}$ demonstrated abnormalities in the vascular architecture associated with malignant breast lesions. Abnormalities included tumor stains, irregular and large caliber vessels, and either prolonged or rapid emptying of contrast agent, presumably owing to leaky vessels and arteriovenous shunts. These findings have been found primarily in relation to malignancy and were relatively absent in benign lesions and very rare in normal patients. More recent preliminary work, using $\mathrm{CT}^{2}$ and digital subtraction angiography, ${ }^{3}$ consistently demonstrated retention of contrast material and abnormal vasculature in malignant breast lesions. Such work suggests that the use of a less invasive technique, such as Doppler ultrasonography, to evaluate flow abnormalities for the purpose of detecting breast cancer could have practical clinical value.

Specific Doppler changes in breast cancer have been widely reported in studies using $10 \mathrm{MHz} C W$ Doppler probes. $^{4-6}$ Such changes have included a multiplicity of vessels with multidirectional flow, resulting in high Doppler power over the whole range of observed Doppler frequencies and flow at relatively high velocities, presumably due to enlarged vessels and arteriovenous 
shunting and relatively high diastolic flow. Although the specificity was high for cancer diagnosis, the sensitivity with any reasonable amount of scanning time and operator training was, or was expected to be, clinically unacceptable for nonpalpable lesions. Jellins began the movement away from a hand-held single beam by using $10 \mathrm{MHz}$ Doppler in combination with imaging on a water path scanner. ${ }^{7}$ This allowed Doppler assessment of lesions identified on the pulse echo images and resulted in $93 \%$ correct identification of previously identified malignant masses. It should be noted, however, that most, if not all, Doppler studies published to date have been on women in whom there was a high suspicion of cancer and the lesions were relatively large. A long discussion of sensitivities and specificities achieved in the various studies is not necessary here, however. Additional developments are clearly needed, as the studies have not been overwhelmingly convincing to the breast care decision makers. Doppler ultrasonography is not used routinely in most breast cancer diagnosis, and gray scale imaging plays primarily a simple role of solid-cyst differentiation. Improvements in detecting tumor vasculature and assessing its morphology, as addressed here, might help expand the role of ultrasonography through more frequent assessment of breast tumor vascularity.

Researchers using color flow imaging have already claimed that the technique is more promising for practical Doppler evaluation of the breast than traditional hand-held $\mathrm{CW}$ images. ${ }^{8-10}$ Our own work ${ }^{89}$ not only indicated a potential role for use of such instruments in the diagnosis of breast cancers but also suggested that with only modest increases in sensitivity, a Doppler flow imager could display the small vessels said to give signals characteristic of malignant lesions. Effects of various vasoconstriction techniques on the flow in these vesseis also were detected. Although Cosgrove and associates ${ }^{10}$ were quite confident about the color flow technique in human breast cancer, our own experience with the same $(5 \mathrm{MHz})$ color flow scanner and a different color flow system with $7.5 \mathrm{MHz}$ Doppler was that the sensitivity to small vessel flow needed to be increased to achieve the excellent results previously reported with $10 \mathrm{MHz} C W$ systems. In addition, considerable difficulties also exist in evaluating small vessel flow quantitatively and objectively from the information usually extractable from two-dimensional color flow images. Sampling at regular intervals in space and in the cardiac cycle requires great care as well. All of these difficulties can be addressed by 3D scanning as we have done here.

It is difficult to assess the shape and extent of vasculature when normal vessels and tumor vessels of similar size are not visualized over much of their length. In most patients, the deeper vessels of normal size are seen only occasionally as small segments, where the signal attenuation due to beam aberrations as well as absorption and diffuse scattering are minimal. Because a CW system could be adapted to fast color flow imaging, ${ }^{11}$ we first performed a comparative study using an older $\mathrm{CW}$ system and a pulsed color flow system to determine whether system signal sensitivity to small breast vessels really was significantly higher with the CW system.

Even when Doppler sensitivity is adequate it often is difficult to fully utilize the shape distortions of tumor neovasculature from normal, particularly if the radiologist is not personally available to perform an extensive color flow examination. Figure 1A shows a typical appearance of a relatively large, superficial, normal mammary vessel captured in longitudinal cross section. It may be distinguished easily from the highly vascular breast cancer depicted in Figure 1B, but not from the vessels of many carcinomas that may be visualized only as one or two small vessels or as a very short length of a vessel. ${ }^{9}$ Such a small vessel segment was the only vasculature observed in a $1 \mathrm{~cm}$ intraductal carcinoma in a different quadrant of the same breast as that depicted in Figure 1A. It was hypothesized that if the breast vasculature could be displayed clearly in $3 \mathrm{D}$, the anatomic pattern of neovasculature would be notably different, both visually and quantitatively, from normal vascular branching patterns. We report here our initial demonstrations of 3D color flow images of small, pulsatile vessels.

\section{METHODS}

\section{CW-Pulsed Wave Sensitivity Comparison}

Six women with palpable breast masses and mammograms suggestive of malignancy were scanned prior to surgical biopsy after obtaining informed consent. The CW-pulsed wave comparison was performed with a Dopscan Model $150 \mathrm{CW}$ Doppler system (Carolina Medical Electronics, King Carolina) employing fixed, hand-held transducers, for a comparison with a color flow Doppler system (Acuson Model 128, Mountain View, CA) using a $7.5 \mathrm{MHz}$ linear array, Model 7384, operated in Doppler mode at $5 \mathrm{MHz}$. All patients were scanned in the supine position, sometimes with the ipsilateral arm raised above the head. Power levels for each examination were noted to be at "standard" (i.e., maximum) power settings. The slow flow setting was employed, and the other usual user settings were adjusted to maximize detection of the small breast vessels. The area of interest was localized by palpation and visualized initially by gray scale, after which the color flow feature was employed for visualization of vessels (peripheral, adjacent, and feeders when possible). 


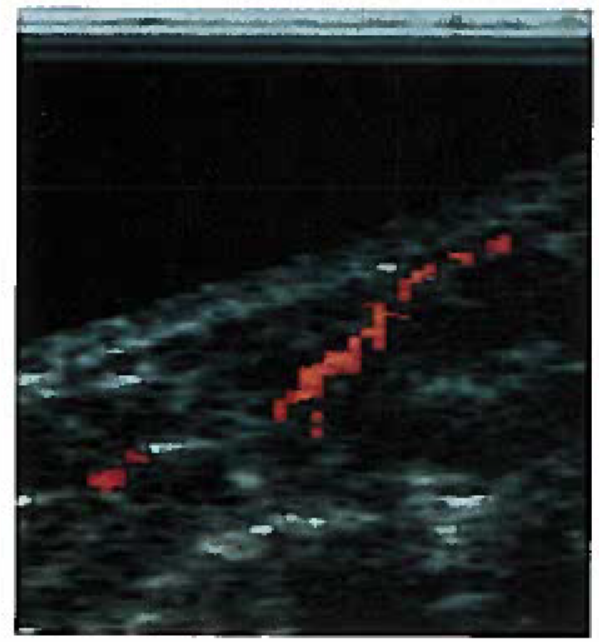

$\mathbf{A}$

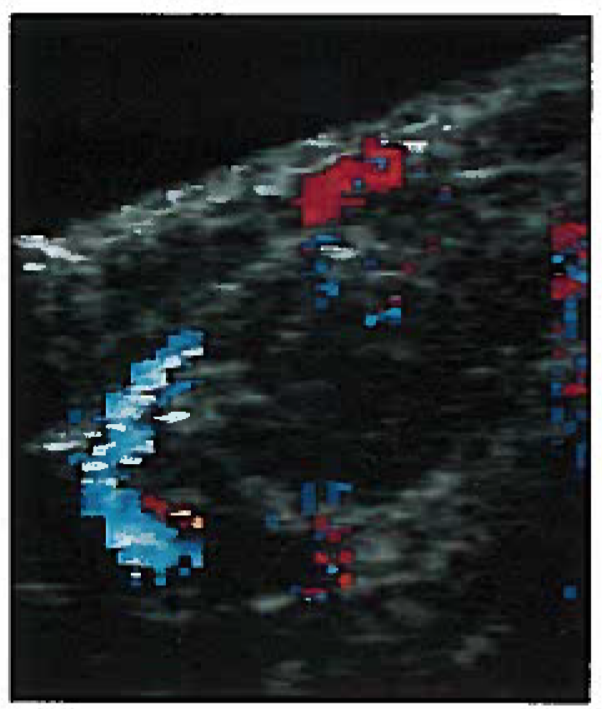

B

Figure 1 Color flow images of breast vasculature. A, Normal appearing superficial vessel. B, Extensive neovasculature around the periphery of a large, infiltrating ductal carcinoma.

Spectra of representative arterial flow were recorded and spectral measurements were performed.

The final portion of the examination was performed with the Dopscan Model $150 \mathrm{CW}$ unit. An angleadjustable transducer pair, depicted in Figure 2, and a Carolina Medical $10 \mathrm{MHz}$ "split D" pencil probe were utilized during the examination. The first portion of the $C W$ examination was performed with the split $D$ probe, followed by examination and spectral recording with the V-shaped angle adjustable pair. All spectra were measured for the various qualitative and quantitative features listed in Table 1. The first four measures are explained adequately in the table. In measures 5 and 6 and elsewhere we use the term "Doppler spectrogram" or "time spectrogram" as a refinement to the usual terminology. A Doppler spectrum should refer to a plot of Doppler signal power as a function of frequency. When signal power is represented by color or gray scale, on a Doppler frequency axis, and those spectra are a function of time along the second axis (as is normally done), that constitutes a time spectrogram or time-frequency distribution.

Figure 2 Transducer holder and arrangement for CW Doppler tomography.
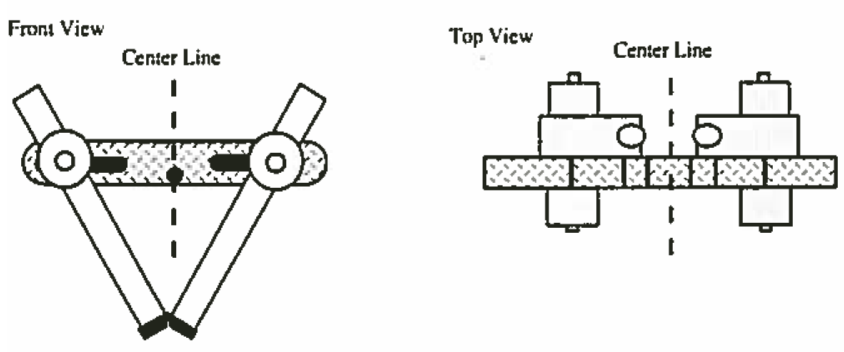

The spectral measures described were chosen to test whether the spectral flow properties reported previously to be characteristic of cancer were present in these $\mathrm{CW}$ studies and to assess whether the analogous measurements on pulsed Doppler spectra would track with the $\mathrm{CW}$ measures in a way that might be indicative of blood flow properties in cancer.

The term sensitivity is not used here in the most rigorous clinical trials definition but rather as system sensitivity represented by small vessel detectability (i.e., the relative number of small vessels, over a range of depths in the breast, which are imageable or yield a spectrum of reasonable signal-to-noise ratio). An overall subjective sensitivity comparison was also given as the system operator's impression of Doppler signal strength and its spatial and temporal extent. These are clinical measures related to the controlled physical measurements of Doppler system sensitivity to small vessel flow and of clutter dynamic range. The former physical measurement provides the system signal-tonoise ratio for a small vessel, with minimal surrounding stationary echoes and obtained as a function of range and overlying attenuation coefficient. The latter gives the maximum ratio of stationary reflection to detectable Doppler signal.

The eighth measurement tabulated in Table 1 was an estimate of whether the power in the spectrum was in a thin curve, as would be expected in a CW spectrogram for uniform (or plug) flow, or whether it was spread from high to minimum frequency shifts, weighted toward the low frequency shifts, as would be expected in CW spectra for sample volumes containing flow in a wide range of directions. Measurement number 9 was very similar, with a value in an ideal spec- 
Table 1: Properties Measured from the Pulsed Doppler Color Flow Images and Spectrograms and the CW Doppler Spectrograms

\begin{tabular}{|c|c|}
\hline Property & Criteria \\
\hline $\begin{array}{l}\text { 1. Number of vessels observed in the area of the } \\
\text { tumor }\end{array}$ & Rated as 0,1 , or multiple \\
\hline 2. Tumor depth & Tumor distance from the skin on the sonographic image \\
\hline 3. Vessel location relative to the tumor & $\begin{array}{l}\text { S, superficial; } L \text {, lateral; } D \text {, distal; } P \text {, posterior; } M \text {, medial; } \\
C \text {, combination or removed from tumor; } N O \text {, not ob- } \\
\text { served }\end{array}$ \\
\hline $\begin{array}{l}\text { 4. Vessel location-relative radial distance from the } \\
\text { tumor center }\end{array}$ & $\begin{array}{l}P \text {, in tumor periphery; } A \text {, adjacent to tumor; } W \text {, well } \\
\text { outside tumor; } I \text {, in tumor core; } N O \text {, not observed }\end{array}$ \\
\hline $\begin{array}{l}\text { 5. Peak systolic Doppler shift (peak value of the } \\
\text { envelope of the absolute value of the Doppler } \\
\text { spectrogram) }\end{array}$ & $\begin{array}{l}\text { Peak systole: } 1,000 \text { times the peak Doppler shift }(f) \text { as a } \\
\text { fraction of the ultrasonic beam center frequency }\left(f_{i}\right)\end{array}$ \\
\hline $\begin{array}{l}\text { 6. Minimum diastolic Doppler shift (minimum value } \\
\text { of the envelope of the absolute value of the } \\
\text { Doppler spectrogram) }\end{array}$ & $\begin{array}{l}\text { Minimum diastole: } 1,000 \text { times the minimum Doppler } \\
\text { shift (f) as a fraction of the ultrasonic beam center } \\
\text { frequency }\left(f_{0}\right)\end{array}$ \\
\hline 7. Resistive index & $\begin{array}{l}\text { Doppler shift ratio: (Peak systole - Minimum diastole)/ } \\
\text { Peak systole }\end{array}$ \\
\hline $\begin{array}{l}\text { 8. Estimated relative power in the high and low } \\
\text { velocity portions of the observed power spec- } \\
\text { trum; when different estimates were obtained } \\
\text { for different vessels, they were reported }\end{array}$ & $\begin{array}{l}\text { 5. Power clearly in the high velocity portion as in uniform } \\
\text { flow; } 4 \text {, power more in high velocity; } 3 \text {, mixed; } 2 \text {, power } \\
\text { more in low velocity; } 1 \text {, power clearly in low velocity } \\
\text { portion of the spectrum, as would be observed, at least } \\
\text { with } \mathrm{CW} \text {, in turbulent or multidirectional flow }\end{array}$ \\
\hline $\begin{array}{l}\text { 9. Largest percentage of spectral energy with either } \\
\text { a positive or a negative frequency shift (polarity) }\end{array}$ & 50 to $100 \%$ \\
\hline $\begin{array}{l}\text { 10. Subjective } C W \text { apparent Doppler sensitivity ver- } \\
\text { sus color flow imaging }\end{array}$ & Graded from 5, Clearly higher to 1, clearly lower \\
\hline $\begin{array}{l}\text { 11. Subjective CW apparent Doppler sensitivity ver- } \\
\text { sus pulsed Doppler spectra }\end{array}$ & 5, Clearly higher; 1 , clearly lower \\
\hline
\end{tabular}

trogram of $100 \%$ if all the flow were away from or toward the transducer and a value of $50 \%$ in a $\mathrm{CW}$ spectrogram if it were split equally in the two directions.

We should note that spectral measurements do not necessarily have the same meaning in pulsed Doppler spectra (actually time spectrograms), as in true CW time spectrograms, in which signal power is represented by brightness or color as a function of frequency and time. In pulsed Doppler spectrograms, multiple samples of the mean frequency are acquired in each time window and plotted as a function of time." The vertical spread of points in any time window represents the variation in the mean frequency shifts, due to electronic and speckle noise and actual changes in average reflector motion in the sample volume over the short time period. Thus, the degree to which historic spectral measures of turbulence and other multidirectional flow from $\mathrm{CW}$ studies can be translated directly to pulsed Doppler studies is not clear to us.

\section{D Color Flow Imaging System}

For 3D color flow mapping of tumor vasculature, a linear array of a different color flow imager (Quantum Quad 1, Siemns Quantum Issaquiah, Washington) was interfaced to a position-encoding scanning arm as illustrated in Figure 3. Both the 7.5 and $5 \mathrm{MHz}$ linear arrays were evaluated. The color flow imager provided a digital image recording mode on its video cassette recorder (VCR). An audio frequency signal was amplitude modulated by potentiometer P1 in proportion to the linear position of the scan head. That signal was

Figure 3 Recording system for color flow images and fouraxis position signals. For these initial studies, only the linear translation signal from potentiometer P1 was recorded, with axes 2 and 4 locked during scanning to maintain parallel slices.

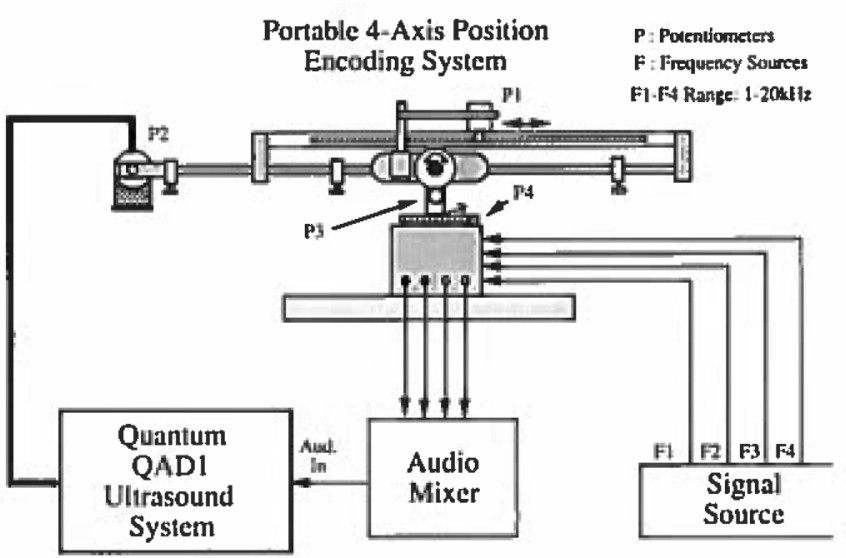


recorded in an audio channel of the VCR. This allowed data acquisition in real time for minimum possible examination time. Essentially linear scanning was employed for these demonstrations, but in the future, the full 3D position and orientation of the scan head can be recorded by applying different frequencies to each of the remaining three scan arm potentiometers and mixing the signals before recording.

Digital transfer of images from the digital video cassette and scanner to a portable, personal computer (PC)-compatible, data acquisition computer was performed at a convenient time after the examination. The off-line processing is diagrammed in Figure 4. The position signal was digitized and monitored by a $\mathrm{Na}$ tional Instruments board and software in the data acquisition computer, and the color flow scanner was automatically frozen in the cine loop mode after the scan plane had moved by a selected distance. The software can resolve the amplitude of four mixed signals by Fourier analysis. Because the linear scans had been performed at a slow rate, less than one half of the expected plane separation per second, an image within a small number of frames of the stopping place could be chosen visually for maximum apparent flow (local systole). The set of images selected during local systole and exhibiting a minimum of random color flow noise were then transferred to a Stellar 3D graphics workstation and displayed by one of several networks programmed in the Advanced Visualization System (AVS). One display method used rays traced through the 3D volume of data at selected orientations. Brightness, hue, saturation, ray attenuation and opacity, and other characteristics were assigned as a function of gray scale pulse echo amplitude and Doppler color (frequency shift signal). A second useful display method combined up to three orthogonal slices through the data set, usually showing the gray scale as well as the color flow data in those planes. Color flow data outside those planes was displayed as a shaded surface

Figure 4 Image and position signal data transfer and display processing systems. Only one readout axis was employed in this study.

Slice Selection

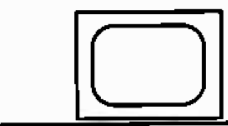

3-D Workstation

with AVS

Ethemet

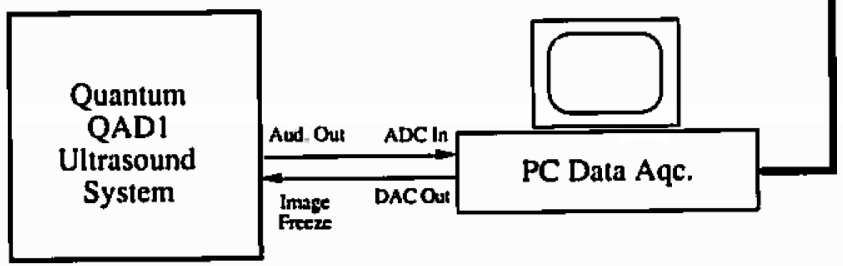

of connected polygons, with no color change for various flow velocities.

The system was evaluated on a test object (Radiation Measurements, Model 425, Middleton, WI), on three normal volunteers, and on one rabbit with a VX-2 carcinoma, consisting of three nodules from 1 to $5 \mathrm{~cm}$ across, located in its thigh.

\section{RESULTS}

\section{CW-Pulsed Wave Sensitivity Comparison}

Mammographic and histologic information for the six patients with carcinoma is summarized in Table 2. Four of the six neoplasms were under $2.5 \mathrm{~cm}$ in diameter. As shown in Table 3, the CW spectroscopy system was observed to be more sensitive to the tumor vasculature for almost all measurements. More areas of flow were observed with $\mathrm{CW}$ (e.g., one or more vessels were observed with $\mathrm{CW}$ in two cases in which color flow imaging revealed no flow near the tumor). The average minimum distance from the skin surface along the path followed for the $C W$ examination was $1 \mathrm{~cm}$. In the three cases in which meaningful pulsed Doppler spectra could be obtained, mean frequency shifts in $\mathrm{kHz}$ per $\mathrm{MHz}$ ultrasound frequency were quite similar for the $\mathrm{CW}$ and the pulsed systems. At the time of peak systolic frequency shift, these ratios (Doppler shift frequency in $\mathrm{kHz}$ per $\mathrm{MHz}$ ultrasound frequency) were 1.76 and 1.63 for $C W$ and pulsed wave, respectively, and 0.60 and 0.60 at the time of minimum diastolic frequency shift. In half the cases, adequate spectra could not be obtained with the pulsed system to make these measurements. The resistive index was essentially the same with both Doppler systems.

Both Doppler systems gave approximately the same medium result ( 3 or 4 ) on measurement 8 . That relative distribution of spectral power between high and low velocities was for the largest areas of flow in the three subjects for whom results could be obtained with the pulsed Doppler. However, in the areas of weaker signal in two of these subjects, and in the other three subjects, the $\mathrm{CW}$ system spectra showed a greater proportion of low frequency shifts. In measurement 9 , the percentage of spectral power in the main polarity, the results with the $\mathrm{CW}$ system uniformly indicated more omnidirectional flow. Overall subjective observations of the ultrasonographer performing the examinations were that the CW system was more sensitive ( 4 on a scale of 5 ) in detecting tumor flow than either the color flow imaging or the pulsed Doppler spectral analysis.

\section{D Color Flow Imaging Results}

The excellent display achieved for pulsatile flow with two different 3D visualization methods is reflected to 
Table 2: Mammographic and Histologic Data for the Six Breast Cancers

\begin{tabular}{|c|c|c|c|c|c|c|c|}
\hline \multicolumn{7}{|c|}{ Mammographic Data } & \multirow[b]{2}{*}{ Final Diagnosis } \\
\hline $\begin{array}{c}\text { Subject } \\
\text { No. }\end{array}$ & $\begin{array}{l}\text { Age } \\
\text { (yr) }\end{array}$ & $\begin{array}{c}\text { Breast } \\
\text { Density }\end{array}$ & $\begin{array}{c}\text { Involved } \\
\text { Breast }\end{array}$ & Tumor Location & $\begin{array}{c}\text { Maximum } \\
\text { Diameter }(\mathrm{cm})\end{array}$ & $\begin{array}{c}\text { Tumor } \\
\text { Calcifications }\end{array}$ & \\
\hline 1 & 71 & Fatty & Left & Upper outer quadrant & 2.5 & Yes & Invasive and intraductal carcinoma \\
\hline 2 & 44 & Mixed & Right & Upper inner quadrant & 4 & No & Invasive and intraductal carcinoma \\
\hline 3 & 48 & Fatty & Right & Upper outer quadrant & 5 & Yes & Invasive/adenocarcinoma \\
\hline 4 & 62 & Mixed & Right & 12 o'clock position & 1.5 & No & Invasive ductal carcinoma \\
\hline 5 & 70 & Fatty & Left & 12 o'clock position & 2.5 & Yes & Intraductal carcinoma \\
\hline 6 & 46 & Mixed & Left & 12 o'clock position & 1 & No & Invasive and intraductal carcinoma \\
\hline
\end{tabular}

some extent in the color photographs in Figures 5 and 6. Even more revealing for images of complex vasculature is rotation of the volumetric display on a monitor. Figure 5 shows two displays of flow data obtained by scanning a Radiation Measurements Model 425 Doppler flow phantom every millimeter with the experimental system described in Figures 3 and 4. Figure 5A is a two-dimensional projection of the 3D data in which the depth cue is obtained by decreasing the gray scale pixel brightness as a function of depth (termed "opacity") along a line of site. The color pixels representing flow are made completely opaque to maintain maximum flow information. The small dropouts in the 8 $\mathrm{mm}$ diameter simulated vessel of the test object prob-

Table 3: Acoustic Data for the Six Breast Cancers

\begin{tabular}{|c|c|c|c|c|c|c|c|}
\hline \multirow{2}{*}{ Property } & \multicolumn{7}{|c|}{ Subject Number } \\
\hline & 1 & 2 & 3 & 4 & 5 & 6 & Mean \\
\hline \multicolumn{8}{|l|}{ Color Flow Pulsed at $5 \mathrm{MHz}$} \\
\hline 1. Number of vessels & Mult & Mult & Mult & Mult & 0 & 0 & - \\
\hline 2. Tumor depth $(\mathrm{cm})$ & 2 & 1 & 0.5 & 1 & 0.5 & 1 & - \\
\hline 3. Vessel location & $S, M$ & S,L,M & S,L,D,M & $S, L$ & NO & No & - \\
\hline 4. Vessel radial distance & $P, A, W$ & P.A & P,A,W & $\mathrm{P}, \mathrm{A}$ & NO & No & - \\
\hline $\begin{array}{l}\text { 5. Peak systole }(1,000 \mathrm{f} / \\
\left.\mathrm{f}_{0}\right)^{*}\end{array}$ & - & 1.73 & 2.21 & 1.35 & - & - & 1.76 \\
\hline $\begin{array}{l}\text { 6. Minimum diastole } \\
\left(1,000 \mathrm{f} / \mathrm{f}_{4}\right)^{*}\end{array}$ & - & 0.74 & 0.77 & 0.29 & - & - & 0.60 \\
\hline 7. Resistive index & - & 0.57 & 0.63 & 0.77 & - & - & 0.66 \\
\hline $\begin{array}{l}\text { 8. Power spectrum at high } \\
\text { or low velocity }\end{array}$ & - & 3 & 3 & $3-4$ & - & - & - \\
\hline $\begin{array}{l}\text { 9. Percentage in main po- } \\
\text { larity }\end{array}$ & - & 90 & 95 & 98 & - & - & 9.4 \\
\hline $\begin{array}{l}\text { 10. CW sensitivity versus } \\
\text { color flow }\end{array}$ & 4 & 4 & 3 & 3 & 5 & 5 & 4.0 \\
\hline \multicolumn{8}{|l|}{$\mathrm{CW}$ at $10 \mathrm{MHz}$} \\
\hline 1. Number of vessels & Mult & Mult & Mult & Mult & Mult & Mult & - \\
\hline 2. Tumor depth $(\mathrm{cm})$ & - & - & - & - & - & - & - \\
\hline 3. Vessel location & $S, M$ & $\mathrm{~S}, \mathrm{~L}, \mathrm{M}$ & $\mathrm{L}, \mathrm{M}$ & S,L & S,L,D,M & $\mathrm{L}$ & - \\
\hline 4. Vessel radial distance & $\mathrm{P}, \mathrm{A}$ & P,A & P,A,W & $\mathrm{P}, \mathrm{A}$ & $\mathrm{P}, \mathrm{A}$ & $\mathbf{P}$ & - \\
\hline $\begin{array}{l}\text { 5. Peak systole }(1,000 \mathrm{f} / \\
\left.f_{0}\right)^{*}\end{array}$ & 0.80 & 1.28 & 2.33 & 1.28 & 1.72 & 1.02 & 1.41 \\
\hline $\begin{array}{l}\text { 6. Minimum diastole } \\
\left(1,000 \mathrm{f} / \mathrm{f}_{0}\right)^{*}\end{array}$ & 0.30 & 0.45 & 1.00 & 0.34 & 0.47 & 0.34 & 0.48 \\
\hline 7. Resistive index & 0.63 & 0.65 & 0.56 & 0.73 & 0.72 & 0.67 & 0.66 \\
\hline $\begin{array}{l}\text { 8. Power spectrum at high } \\
\text { or low velocity }\end{array}$ & 1 & 3,2 & $4,3,2$ & 3 & 1 & 2 & 2.3 \\
\hline $\begin{array}{l}\text { 9. Percentage in main po- } \\
\text { larity }\end{array}$ & & 60 & 71 & 63 & 54 & 60 & 6.5 \\
\hline $\begin{array}{l}\text { 11. CW sensitivity versus } \\
\text { pulsed Doppler spectra }\end{array}$ & & - & 3 & 3 & 5 & 5 & 4.2 \\
\hline
\end{tabular}

- $f$ and $f_{0}$ are the Doppler and center frequencies, respectively.

Dashes $=$ not available. 


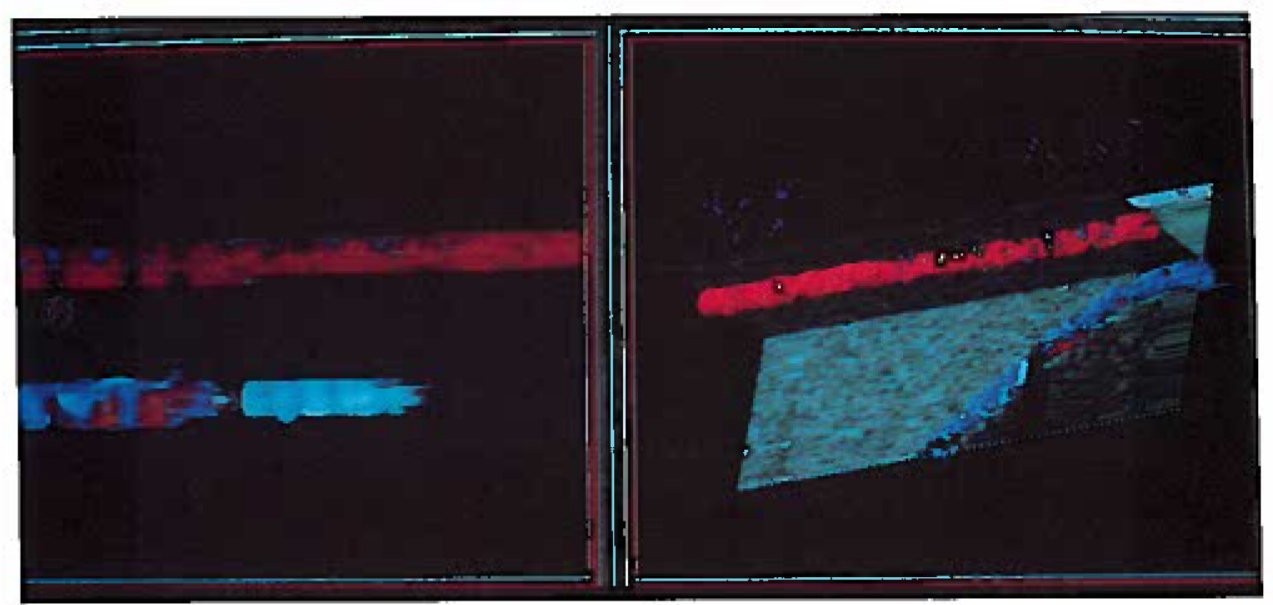

A

B

Figure 5 Three-dimensional representations of images taken of a Doppler flow phantom using a two-dimensional projection with opacity shaded gray scale (A) and an isosurface rendering of flow with orthogonal gray scale slices (B).

ably would be eliminated by operating the scanner in a peak-detect (persistence) mode with appropriate integration time. This should usually be worthwhile even though noise would be increased somewhat.

Figure $5 B$ is obtained using an isosurface rendering in which transitions between gray scale and color are used to define points on a color surface and artificial shading is added as a depth cue. In addition, orthogonal slices are selected from within the data set to present the gray scale information from those planes. The predominantly blue flow is in a simulated vessel whose depth is changing within the phantom. A constriction in this tube is revealed by a shadow in the pulse echo plane that approximately bisects the vessel. Note also that in Figure 5A this constriction can be seen as a narrowing of the tube along with turbulence or recirculation to the left of the constriction displayed as a mix of red and blue colors. The color scale in

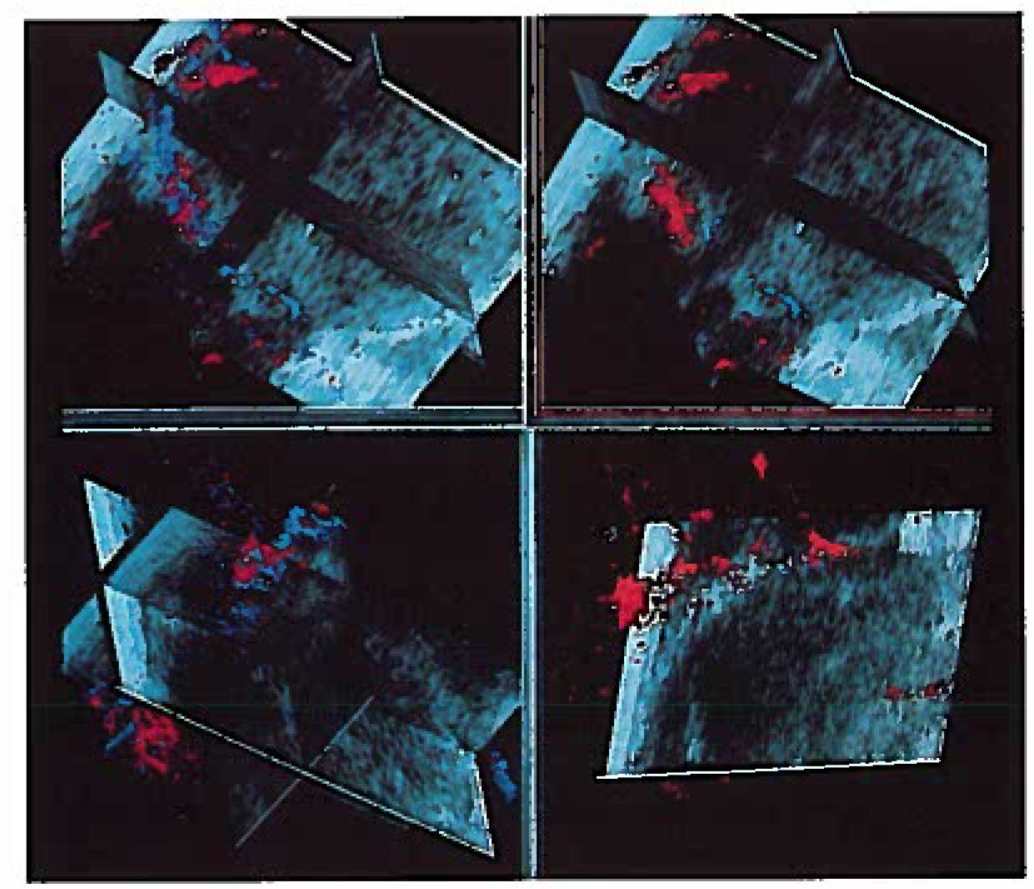

Figure 6 Three-dimensional images of a VX2 carcinoma in a $5 \times 6 \times 5 \mathrm{~cm}$ volume of a rabbit thigh. All out-of-plane, blue coded color has been removed from the images on the right to clarify vascularity of a given flow direction. In the lower right image, two of the orthogonal planes have been removed to reveal several adjacent pixel groupings, such as the one indicated by the pointer, which represent vessels whose path probably would not often be delineated without 3D display. 
Figure 5A goes from dark blue to blue-white and from dark red to red-white for low to high positive and negative Doppler shifts, respectively. Fairly high Doppler shifts are shown in the constriction and to the right of it. The relative lack of high frequency shifts to the left of the constriction may be due to the color flow imager displaying each sample volume's mean frequency shift in the turbulent flow region to the left of the constriction. This frequency shift information is not presented in the threshold-detected isosurface rendering of Figure 5B.

The isosurface rendering technique is used again in Figure 6 with a variety of viewing angles and with differing amounts of information displayed. This figure of a rabbit VX-2 carcinoma in the thigh is particularly impressive, not only for the clear representation of the overall vascular anatomy but also for the display of small vessels as sparse, curvilinear traces of pixels, vessels whose orientations usually would not be revealed in conventional color flow images. This example might be more impressive if a larger area had been scanned, showing the vessel geometry coming into this complex shell of vessels.

\section{DISCUSSION AND CONCLUSIONS}

The detection of an increased number of small vessels with the $C W$ system and the operator's subjective impression of increased $\mathrm{CW}$ flow detection sensitivity compared with either the color flow image or pulsed Doppler spectrum was consistent with results in a recent paper ${ }^{12}$ comparing a hand-held $\mathrm{CW}$ system with a color flow imager. In addition to allowing detection of more vessels or flow locations, the CW system appeared to be more sensitive in measurements 8 (breadth of the Doppler spectrum) and 9 (bidirectionality) with respect to the type of small-vessel, multidirectional flow loci reported to be characteristic of breast cancer. Increased bidirectionality in cancer was observed by White and Cledgett ${ }^{13}$ but not by Burns ${ }^{4}$ or Madjar et al. ${ }^{12}$ The relative $\mathrm{CW}$-pulsed wave sensitivity to multidirectional flow could also be the result of the extended sample volume with the $\mathrm{CW}$ transducer system, even with the crossed transmitter and receiver beam patterns. A reduction in resistive index, which might have been expected for the small-vessel cancer signals, was not seen. Similarly, Madjar and coworkers ${ }^{12}$ did not see a change in the systolic-diastolic frequency shift quotient and Burns and colleagues $^{4}$ did not see a change in pulsatility index between normal and malignant breast vasculature. This degree of inconsistency in the literature suggests that the tumor characteristics easily observable with available equipment are not entirely consistent or charac- teristic themselves. However, we believe that visualization and characterization of vascular abnormalities or of the vascularity of suspected lesions might well prove useful eventually in breast cancer diagnosis and even detection.

This small, preliminary $\mathrm{CW}$-pulsed wave comparison is valuable mainly as an incentive for a larger study or direct development of a CW scanning system that can conveniently and rapidly be used to study suspected regions in the breast at higher flow detection sensitivity. This would allow more meaningful clinical trials of tradeoffs between sensitivity and spatial resolution. Range resolution in the CW scanner would be provided by crossing the transmit and receive beams. ${ }^{14}$

Three-dimensional display of the color flow data appears promising for display of the vascular anatomy to aid visual detection of abnormal vascular morphology. In a hypervascular case, as in Figures $1 \mathrm{~B}$ and 6, there is no question that a lesion with abnormal vascularity is present during color flow scanning or from two-dimensional color images therefrom. In Figure 6, small traces of color dots are seen that, in 3D, may be observed curving around the lesion and that could not be easily linked to each other in the color flow digital videotapes as representing the same vessel. This improved detectability of small vessel structure should aid diagnosis in some cases. Having the pulse echo slice information combined with the vascular morphology also provides increased confidence that a vascular anomaly is associated with a lesion and not just dysplasia. However, we suspect also that some of the main, normal vessels in a significant fraction of human breasts are probably below the threshold for consistent color flow display of their morphology by current color flow scanners with any display method. Therefore, if the sonographer wishes to detect structural deviations that would occur in the vicinity of many small lesions, an improvement in the Doppler sensitivity would probably be required to make an effectively complete mapping of the vascular tree. It remains to be determined how many cases are in each category, whether Doppler sensitivity can be improved electroacoustically or with contrast agents, and what the role of vessel morphology will be, given the promise of fast, inexpensive examinations.

Storage of the data slices in their true 3D orientation also will allow more accurate quantitative analysis of the vascularity, from simple volume fractions of detected flow as described by Cosgrove and associates, ${ }^{10}$ to more complex morphologic measures such as the fractile dimension of the vasculature. ${ }^{15}$ There are some advantages to real time imaging of vascularity in two dimensions compared with a typical 3D volume set. The human observer, if patient, can filter real flow signals from noise by their temporal behavior. Three- 
dimensional processing with most available equipment also is slow at present. It might be expected that most of the limitations of 3D display can be overcome with advances in visualization science and development of specific applications.

\section{REFERENCES}

1. Feldman F, Habif D, Fleming RJ, et al: Arteriography of the breast. Radiology 89:1053, 1967

2. Chang CHJ, Nesbit DE, Fisher DR, et al: Computer tomographic mammography using a conventional body scanner. AJR 138:553, 1982

3. Flynn MJ, Carson PL, Meyer CR: Quantitative ultrasonic imaging in medical diagnosis. Med Information Processing 2:42, 1984

4. Burns PN, Halliwell M, Wells PNT: Ultrasonic Doppler studies of the breast. Ultrasound Med Biol 8:127, 1982

5. Minasian H, Bamber JC: A preliminary assessment of an ultrasonic Doppler method for the study of blood flow in human breast cancer. Ultrasound Med Biol 4:357, 1982

6. Boyd J, Jellins J, Reeve TS: Doppler examination of the breast. In Jellins J, Kobayashi P (eds): Ultrasonic Examination of the Breast. New York, John Wiley \& Sons, 1983, p 385
7. Jellins J: Combining imaging and vascularity assessment of breast lesions. Ultrasound Med Biol 14:121, 1988

8. Rubin JM, Carson PL, Zlotecki RA: Visualization of tumor vascularity in a rabbit VX2 carcinoma by Doppler flow mapping. J Ultrasound Med 5:569, 1987

9. Adler DD, Carson PL, Rubin JM, et al: Doppler ultrasound color flow imaging in the diagnosis of breast cancer: Preliminary findings. Ultrasound Med Biol $16: 553,1990$

10. Cosgrove DO, Bamber JC, Davey JB: Color Doppler signals from breast tumors (work in progress). Radiology 176:175, 1990

11. Carroll BA, von Ramm OT: Fundamentals of current Doppler technology. Ultrasound Q 6:275, 1988

12. Madjar $\mathrm{H}$, Sauerbrie W, Munch S: Continuous-wave and pulsed Doppler studies of the breast: Clinical results and effect of transducer frequency. Ultrasound Med Biol 17:31, 1991

13. White DN, Cledgett PR: Breast carcinoma delection by ultrasonic Doppler signals. Ultrasound Med Biol 4:329. 1978

14. Riley JK, Miller-Jones SM: Method of CW Doppler imaging using variably focused ultrasonic transducer array (General Electric). U. S. Patent No. 4,598,589, 1986

15. Honda $E$, Domon $M$, Sasaki T: A method for determination of fractal dimensions of sialographic images. Invest Radiol 26:894, 1991 